\title{
Soluble HLA-DR serum levels are associated with smoking but not with acute coronary syndrome
}

\section{Tolva, Johanna}

\section{7}

Tolva , J , Paakkanen , R , Jarva , H , Pussinen , P , Havulinna , A S , Salomaa , V , Sinisalo , J \& Lokki , M-L 2017 , ' Soluble HLA-DR serum levels are associated with smoking but not with acute coronary syndrome ' , Atherosclerosis , vol. 266 , pp. 58-63 . https://doi.org/10.1016/j.atherosclerosis.201

http://hdl.handle.net/10138/298053

https://doi.org/10.1016/j.atherosclerosis.2017.09.023

publishedVersion

Downloaded from Helda, University of Helsinki institutional repository.

This is an electronic reprint of the original article.

This reprint may differ from the original in pagination and typographic detail.

Please cite the original version. 


\title{
Soluble HLA-DR serum levels are associated with smoking but not with acute coronary syndrome
}

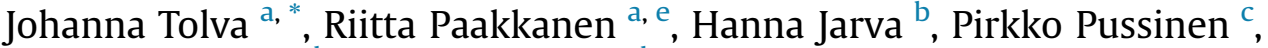 \\ Aki S. Havulinna ${ }^{d}$, Veikko Salomaa ${ }^{d}$, Juha Sinisalo ${ }^{e}$, Marja-Liisa Lokki a \\ a Transplantation Laboratory, University of Helsinki, Helsinki, Finland \\ ${ }^{\mathrm{b}}$ Department of Bacteriology and Immunology and Research Programs Unit, University of Helsinki, and HUSLAB, Helsinki, Finland \\ ${ }^{\mathrm{c}}$ Department of Oral and Maxillofacial Diseases, University of Helsinki and Helsinki University Hospital, Helsinki, Finland \\ d National Institute for Health and Welfare, Helsinki, Finland

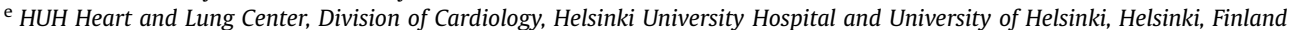

\section{A R T I C L E I N F O}

\section{Article history:}

Received 17 May 2017

Received in revised form

17 September 2017

Accepted 19 September 2017

Available online 21 September 2017

\section{Keywords:}

Coronary artery disease

Acute coronary syndrome

Sandwich ELISA

Soluble HLA-DR

Cigarette smoking

\begin{abstract}
A B S T R A C T
Background \& aims: Elevated soluble HLA-DR (sHLA-DR) serum levels have been reported in HLA class IIassociated inflammatory disorders. We have previously shown that the HLA class II allele HLA-DRB $1^{*} 01$ may predispose to acute coronary syndromes (ACS). To our knowledge, sHLA-DR serum levels have not been studied in ACS.

Methods: sHLA-DR serum levels were measured in 477 ACS patients as cases and 475 area- and sexmatched controls by sandwich enzyme-linked immunosorbent assay. Binary logistic regression and ordinal logistic regression analyses adjusted for clinical parameters were conducted to evaluate the associations of sHLA-DR levels.

Results: ACS patients had lower sHLA-DR serum levels compared to controls ( $\mathrm{OR}=0.837 ; 95 \% \mathrm{CI}=0.704$ $-0.994 ; p=0.043$ ). After adjustment for smoking status, this association was no longer significant. This was explained by the notion that current smoking was inversely associated with sHLA-DR levels both in cases $(\mathrm{OR}=0.592 ; 95 \% \mathrm{Cl}=0.553-0.908 ; p=0.016)$ and in controls $(\mathrm{OR}=0.356 ; 95 \% \mathrm{CI}=0.226-0.563$; $p=0.000010)$. A similar effect was not seen with other cardiovascular risk factors.

Conclusions: The results indicate, for the first time, that lower sHLA-DR levels are associated with smoking, but not with ACS. This is an important finding because previous studies of sHLA-DR have not accounted for the possible associations between smoking and SHLA-DR levels. Further studies are required to confirm these novel results and explore the mechanisms behind the observed associations.
\end{abstract}

(C) 2017 Elsevier B.V. All rights reserved.

\section{Introduction}

Human leukocyte antigen (HLA)-DR molecules are present on the cell surface of antigen-presenting cells and can trigger immune reactions by presenting antigens to $\mathrm{CD} 4$ positive T-lymphocytes. HLA-DR molecules are also found in a soluble form (sHLA-DR) in almost every human body fluid including saliva, sweat, tears, serum, synovial fluid and urine [1-3]. sHLA-DR correlates with the amount of expression of membrane bound HLA-DR, possibly reflecting the inflammatory status [4].

\footnotetext{
* Corresponding author. Transplantation laboratory, University of Helsinki, Haartmaninkatu 3, 00290 Helsinki, Finland.

E-mail address: johanna.m.tolva@gmail.com (J. Tolva).
}

According to previous studies, sHLA-DR serum or plasma levels have been reported to be elevated in patients with inflammatory diseases such as acute phase of multiple sclerosis [5], rheumatoid arthritis [6] and autoimmune hepatitis [7]. In patients with rheumatoid arthritis, sHLA-DR serum levels also correlated with disease activity [8] and decreased after immunosuppressive therapy with cyclosporine A [9]. On the other hand, decreased sHLA-DR serum levels have been associated with end-stage heart disease [10] and severe sepsis [11], and in patients with melanoma, sHLA-DR serum levels declined as the cancer progressed [12].

Disorders showing increased sHLA-DR serum levels have mostly been reported to be HLA class II-associated inflammatory disorders. HLA-DR-positive lymphocytes have been associated with acute coronary syndrome (ACS) [13] and we have shown that the HLA class II allele $H L A-D R B 1^{*} 01$ may predispose to ACS $[14,15]$. In this 
study, we investigated the relationship between sHLA-DR serum levels, ACS and cardiovascular risk factors.

\section{Materials and methods}

\subsection{Study subjects}

We selected ACS patients from the COROGENE study cohort of 5000 patients undergoing coronary angiogram at the Helsinki University Hospital in Finland between June 2006 and March 2008 [16]. Inclusion criteria in the current study were i) ACS, ii) age under 55 years for men and under 65 years for women, iii) home address in the capital region in Finland, yielding 488 cases in total. After excluding the cases with missing serum samples, the group of cases consisted of 477 ACS patients.

475 study subjects from the FINRISK 2007 study cohort served as sex- and area-matched controls [17]. The controls had to be free of cardiovascular disease at baseline. Details of the study subject selection and baseline measurements are reported in the Supplementary data.

All the study subjects gave their signed informed consent. The study protocol conforms to the ethical guidelines of the 1975 Declaration of Helsinki and the Ethics Committee of the University of Helsinki approved the COROGENE study and the Coordinating Ethical Committee of the Helsinki and Uusimaa Hospital District approved the FINRISK study.

\subsection{Quantification of soluble HLA-DR}

We detected sHLA-DR serum levels by sandwich enzyme-linked immunosorbent assay (ELISA) method developed in-house, described in detail in the Supplementary data.

\subsection{HLA-DRB $1^{*} 01$ analyses}

HLA-DRB $1^{*} 01$ genotypes from cases and controls were analysed from genomic DNA by real-time quantitative polymerase chain reaction technique with sequence specific primers as previously described [18]. Genotyping was unsuccessful in 1 case sample and 3 control samples. HLA-DRB $1^{*} 01$ carrier status was considered positive with 1 or 2 allele copies of $H L A-D R B 1^{*} 01$.

\subsection{Analyses for other parameters}

All laboratory parameters: C-reactive protein (CRP), total leukocyte count (TLC), total cholesterol (TC), high-density lipoprotein-cholesterol (HDL-C), low-density lipoprotein-cholesterol (LDL-C), triglycerides (TG) and troponin T (TnT) were defined according to the laboratory standards of HUSLAB (Helsinki University Hospital Laboratory).

\subsection{Statistical analyses}

All the statistical calculations were performed with SPSS 21.0 (SPSS Inc., Chicago, IL, USA). We considered results significant at two-tailed $p<0.05$ not correcting for multiple comparisons. SHLADR levels in serum samples were analysed both as a continuous variable and as a categorical variable as described in detail below. sHLA-DR levels under the detection limit $(<0.1 \mu \mathrm{g} / \mathrm{ml})$ were considered as $0.05 \mu \mathrm{g} / \mathrm{ml}$ when analysing the continuous SHLA-DR variable. sHLA-DR levels and other continuous variables were not normally distributed by the Kolmogorov-Smirnov normality test and logarithmic transformation did not transform them as such, apart from LDL-C and TC levels in controls and HDL-C in cases and controls. All the continuous variables are expressed as mean \pm standard deviation (SD) and categorical variables as count (percentage). We compared continuous variables with a MannWhitney $U$ test, Kruskal-Wallis test and Jonckheere-Terpstra test for trend, which are independent of distributional assumptions. Correlations between clinical parameters and sHLA-DR levels were analysed by nonparametric Spearman's rank-order correlation test.

Based on the visual inspection of the distribution of the continuous sHLA-DR-variable, we cathegorized sHLA-DR levels into four groups: (I) under the detection limit of $0.100 \mu \mathrm{g} / \mathrm{ml}$, (II) $0.100-0.300 \mu \mathrm{g} / \mathrm{ml}$, (III) $0.301-2.000 \mu \mathrm{g} / \mathrm{ml}$ and (IV) over $2.000 \mu \mathrm{g} /$ $\mathrm{ml}$, to obtain a categorical variable of sHLA-DR levels. A Chi-square test, a Mantel-Haenszel Linear-by-Linear Association test for trends or Fisher's exact test was used to determine associations between categorical variables. We compared cases and controls regarding logarithmically transformed sHLA-DR levels in binary logistic regression models, firstly adjusted for age and sex, secondly adjusted for age, sex and smoking status, thirdly in a multivariableadjusted model (adjusted for sex and parameters differing between cases and controls: age, body mass index [BMI], smoking status, diabetes, asthma, cancer and CRP), and finally in a multivariableadjusted model without smoking status.

We performed ordinal logistic regression models adjusted for age and sex using categorical sHLA-DR as a dependent variable, and BMI, smoking status (current versus never-smoking), diabetes, $H L A-D R B 1^{*} 01$, asthma, cancer, rheumatoid arthritis or CRP as explanatory variables, first separately and then together in the same model, to assess the influences of different cardiovascular risk factors on the sHLA-DR levels, determining OR and 95\% confidence intervals (95\% CIs) for ordinal logistic regression models. This OR for a specific explanatory variable measures the odds of being in a group of a higher sHLA-DR level (higher group defined as any of the three separate ways: group IV vs. groups I-III, groups III and IV vs. I and II and groups II-IV vs. group I) compared to the groups of lower sHLA-DR level. Proportional odds of ordinal regression analyses met the criteria for $p>0.050$.

\section{Results}

\subsection{Baseline characteristics}

Baseline characteristics are shown in Table 1. As expected, the cases had more cardiovascular risk factors and cardiovascular medications. Among cases, the distribution of different ACS types was the following: unstable angina $8.2 \%(\mathrm{n}=39)$, ST elevation myocardial infarction $47.6 \%(\mathrm{n}=227)$ and non-ST elevation myocardial infarction $44.2 \%(n=211)$.

\section{2. sHLA-DR levels}

Table 2 presents the sHLA-DR serum levels in the study subjects. Cases had significantly lower levels of sHLA-DR (mean $1.71 \pm 9.53 \mu \mathrm{g} / \mathrm{ml}$ ) than controls (mean $2.83 \pm 17.11 \mu \mathrm{g} / \mathrm{ml}$; $p=0.032$ ). The linear trend was borderline insignificant $(p=0.050)$, showing lower sHLA-DR levels in cases than controls. However, sHLA-DR levels over the 95 th percentile $(>6.67 \mu \mathrm{g} / \mathrm{ml})$ of the control group were found with a similar frequency in cases ( $\mathrm{n}=23,4.8 \%)$.

In the binary logistic regression analysis adjusted for age and sex, the logarithmically transformed sHLA-DR level associated inversely with ACS (OR $=0.811 ; 95 \% \mathrm{CI}=0.681-0.966, p=0.019)$. In the multivariable-adjusted binary logistic regression, the association was no longer significant $(\mathrm{OR}=0.938 ; 95 \% \mathrm{CI}=0.764-1.152$, $p=0.497$ ), indicating that differences in sHLA-DR levels between cases and controls were not explained by disease status, but by other factors differing between cases and controls (Table 3). We 
Table 1

Baseline characteristics.

\begin{tabular}{|c|c|c|c|}
\hline Characteristic & Cases & Controls & $p$-value \\
\hline $\mathrm{n}$ & 477 & 475 & \\
\hline Female & $167(35.0)$ & $169(35.6)$ & 0.854 \\
\hline Age (years) & $50.7 \pm 7.0$ & $53.0 \pm 10.7$ & $<0.001$ \\
\hline Age range (years) & $26.6-64.7$ & $24.4-71.0$ & \\
\hline BMI $\left(\mathrm{kg} / \mathrm{m}^{2}\right)$ & $28.5 \pm 5.7(n=472)$ & $27.3 \pm 4.6$ & $<0.001$ \\
\hline \multicolumn{4}{|l|}{ Risk factors } \\
\hline \multicolumn{4}{|l|}{ Smoking status } \\
\hline Never-smoker & $106(22.2)$ & $235(49.5)$ & $<0.001$ \\
\hline Current smoker & $287(60.2)$ & 109 (22.9) & $<0.001$ \\
\hline Ever-smoker (current + ex) & $371(77.8)$ & $240(50.5)$ & $<0.001$ \\
\hline Diabetic & $66(13.8)$ & $38(8.0)$ & 0.004 \\
\hline Hypertensive & $287(60.2)$ & $273(57.5)$ & 0.398 \\
\hline HLA-DRB $1 * 01$ positive & $169(35.5)$ & $129(27.3)$ & 0.007 \\
\hline \multicolumn{4}{|l|}{ Comorbidities } \\
\hline Asthma & $25(5.2)$ & $45(9.5)$ & 0.012 \\
\hline Cancer & $11(2.3)$ & $26(5.5)$ & 0.011 \\
\hline Rheumatoid arthritis & $20(4.3)$ & $12(2.5)$ & 0.154 \\
\hline \multicolumn{4}{|l|}{ Laboratory parameters } \\
\hline CRP $(\mathrm{mg} / \mathrm{l})$ & $14.8 \pm 27.2$ & $2.5 \pm 5.5$ & $<0.001$ \\
\hline TLC $(10 \mathrm{E} 9 / \mathrm{ml})$ & $10.1 \pm 4.0(\mathrm{n}=460)$ & - & - \\
\hline $\mathrm{TC}(\mathrm{mmol} / \mathrm{l})$ & $4.7 \pm 1.2(\mathrm{n}=399)$ & $5.4 \pm 0.9$ & $<0.001$ \\
\hline HDL-C (mmol/l) & $1.2 \pm 0.4(\mathrm{n}=401)$ & $1.4 \pm 0.4$ & $<0.001$ \\
\hline LDL-C (mmo/l) & $2.7 \pm 1.0(\mathrm{n}=371)$ & $3.3 \pm 0.8$ & $<0.001$ \\
\hline $\mathrm{TG}(\mathrm{mmol} / \mathrm{l})$ & $1.9 \pm 1.7(\mathrm{n}=403)$ & $1.5 \pm 0.9$ & $<0.001$ \\
\hline TnT (ng/l) & $2.7 \pm 4.2(\mathrm{n}=385)$ & - & - \\
\hline \multicolumn{4}{|l|}{ Medication } \\
\hline$\beta$-blockers & $144(62.3)$ & $77(16.2)$ & $<0.001$ \\
\hline ACE inhibitors & $69(37.7)$ & $43(9.1)$ & $<0.001$ \\
\hline ATR blockers & $42(25.9)$ & $34(7.2)$ & $<0.001$ \\
\hline Diuretic & $24(14.5)$ & $27(5.7)$ & $<0.001$ \\
\hline Lipid lowering therapy & $129(60.6)$ & $77(16.2)$ & $<0.001$ \\
\hline
\end{tabular}

Data is presented as mean \pm SD or number of study subjects (\%).

BMI, body mass index; CRP, C-reactive protein; TLC total leukocyte count; TC, total cholesterol; HDL-C, high-density lipoprotein-cholesterol; LDL-C, low-density lipoprotein-cholesterol; TG, triglycerides; TnT, troponin T; ACE, angiotensin-converting enzyme; ATR, angiotensin receptor.

$H L A-D R B 1 * 01$ positive $=1$ or 2 allele copies of $H L A-D R B 1 * 01$.

Data of BMI, TLC, TC, HDL-C, LDL-C, TG, TnT, $\beta$-blockers, ACE inhibitors, ATR blockers, diuretic and lipid lowering therapy were missing from some of the cases.

found that the presence of smoking status changed the significance of the models, adjustment for smoking status reduced the association between sHLA-DR levels and ACS (Table 3). However, the interaction term between smoking status and logarithmically transformed sHLA-DR level was insignificant in relation to ACS patient status, in the binary logistic regression model using ACS patient status as a dependent variable, and logarithmically transformed sHLA-DR level, smoking status and interaction term between smoking status and logarithmically transformed sHLA-DR level as explanatory variables.

\section{3. $S H L A-D R$ levels and cardiovascular risk factors}

Next, we sought to assess the correlation of sHLA-DR and cardiovascular risk factors. In both cases and controls, sHLA-DR levels correlated inversely with current smoking (rho $=-0.112, p=0.026$ and rho $=-0.241, p<0.0001$, respectively). In cases, no other correlations were found, whereas in controls sHLA-DR levels also correlated inversely with age (rho $=-0.111, p=0.016$ ) and HLA$D R B 1^{*} 01$ positivity (rho $=-0.110, p=0.017$ ). The only factor with a significant positive correlation with sHLA-DR was the history of rheumatoid arthritis in controls (rho $=0.153, p=0.001$ ). The Spearman's rank-order correlations between sHLA-DR levels and different variables are summarized in Supplementary Table 1.

When the different sHLA-DR groups (I-IV) were compared against the risk factors, current smoking showed a significant linear trend with decreasing sHLA-DR levels in both cases and controls and rheumatoid arthritis showed a linear trend with increasing sHLA-DR levels in controls (Supplementary Table 2A and B).

We assessed the associations between groups of sHLA-DR level with cardiovascular risk factors by ordinal logistic regression analysis adjusted for age and sex. The results showed that current smoking was inversely associated with sHLA-DR levels in cases and controls (OR $=0.592 ; 95 \% \mathrm{CI}=0.553-0.908 ; p=0.016$ and $\mathrm{OR}=0.356 ; 95 \% \mathrm{CI}=0.226-0.563 ; p=0.000010)$. In addition, HLA$D R B 1^{*} 01$ positivity was inversely and rheumatoid arthritis directly associated with sHLA-DR levels in controls (OR $=0.595 ; 95 \%$ $\mathrm{CI}=0.401-0.882 ; p=0.010$ and $\mathrm{OR}=5.865 ; 95 \%$ $\mathrm{CI}=2.042-16.861 ; p=0.001)$. Multivariable ordinal logistic regression model adjusted for age and sex, including BMI, smoking status (current versus never-smoking), diabetes, HLA-DRB1*01, asthma, cancer, rheumatoid arthritis and CRP as explanatory variables, did not change these results.

Number of cigarettes smoked per day and duration of smoking cessation data were available only for controls and these data were not associated with sHLA-DR levels. Table 4 summarizes the differences in clinical variables between current smokers and neversmokers among cases and controls.

\section{Discussion}

In this study, we discovered a previously unreported association between smoking and lower sHLA-DR levels. This association was confounding our preliminary observation, which showed that ACS patients had lower sHLA-DR levels than controls. As the case population included more smokers than the control population, after correcting for smoking status, no association between sHLADR levels and ACS patient status could be seen. This finding raises the question whether possible associations between smoking and sHLA-DR levels should always be considered when analysing SHLA-

Table 2

Soluble HLA-DR serum levels in study subjects.

\begin{tabular}{|c|c|c|c|c|}
\hline sHLA-DR serum levels & Cases & Controls & $p$-value & $p$-value for trend ${ }^{c}$ \\
\hline sHLA-DR level, mean ( $\mu \mathrm{g} / \mathrm{ml})$ & $1.71 \pm 9.53$ & $2.83 \pm 17.11$ & $0.032^{\mathrm{a}}$ & \\
\hline sHLA-DR level, range $(\mu \mathrm{g} / \mathrm{ml})$ & $0.05-161.77$ & $0.05-268.43$ & & \\
\hline Log-transformed sHLA-DR level, mean (ng/ml) & $2.18 \pm 0.72$ & $2.28 \pm 0.77$ & $0.032^{\mathrm{a}}$ & \\
\hline Log-transformed sHLA-DR level, range $(\mathrm{ng} / \mathrm{ml})$ & $1.70-5.21$ & $1.70-5.43$ & & \\
\hline sHLA-DR groups, $\mathrm{n}(\%)$ & & & & 0.050 \\
\hline$<0.100 \mu \mathrm{g} / \mathrm{ml}(\mathrm{I})$ & $270(56.6)$ & $242(50.9)$ & $0.080^{\mathrm{b}}$ & \\
\hline $0.100-0.300 \mu \mathrm{g} / \mathrm{ml}(\mathrm{II})$ & $92(19.3)$ & $90(18.9)$ & $0.894^{\mathrm{b}}$ & \\
\hline $0.301-2.000 \mu \mathrm{g} / \mathrm{ml}(\mathrm{III})$ & $68(14.3)$ & $88(18.5)$ & $0.075^{\mathrm{b}}$ & \\
\hline$>2.000 \mu \mathrm{g} / \mathrm{ml}(\mathrm{IV})$ & $47(9.9)$ & $55(11.6)$ & $0.389^{\mathrm{b}}$ & \\
\hline
\end{tabular}

\footnotetext{
a Mann-Whitney $U$ test.

b Pearson Chi-square test.

c Mantel-Haenszel Linear-by-Linear Association test for trend.
} 
Table 3

Binary logistic regression models of the associations between logarithmically transformed sHLA-DR levels and ACS patients.

\begin{tabular}{|c|c|c|c|c|}
\hline & \multicolumn{4}{|c|}{ ACS patient status } \\
\hline & \multirow[t]{2}{*}{$p$-value } & \multirow[t]{2}{*}{ OR } & \multicolumn{2}{|c|}{$95 \% \mathrm{CI}$} \\
\hline & & & Lower & Upper \\
\hline \multicolumn{5}{|l|}{ Logarithmically transformed sHLA-DR level } \\
\hline Age and sex adjusted & 0.019 & 0.811 & 0.681 & 0.966 \\
\hline Age, sex and smoking status adjusted & 0.292 & 0.906 & 0.755 & 1.088 \\
\hline Multivariable adjusted ${ }^{a}$ & 0.540 & 0.938 & 0.764 & 1.152 \\
\hline Multivariable adjusted without smoking status ${ }^{\mathrm{b}}$ & 0.047 & 0.820 & 0.673 & 0.997 \\
\hline
\end{tabular}

ACS, acute coronary syndrome.

$\mathrm{OR}$, odds ratio; $\mathrm{CI}$, confidence interval.

a Adjusted for sex and baseline characters differing between cases and controls: age, BMI, smoking status (never-versus ever-smoker), diabetes, $H L A-D R B 1{ }^{*} 01$ carrier status, cancer, asthma and CRP.

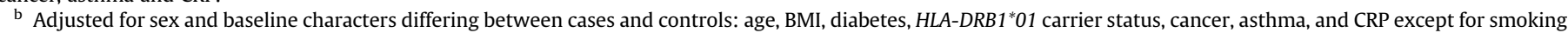
status (never- versus ever-smoker).

Table 4

Differences in clinical parameters between current smokers and never-smokers among cases and controls.

\begin{tabular}{|c|c|c|c|c|c|c|}
\hline & \multicolumn{3}{|l|}{ Cases } & \multicolumn{3}{|l|}{ Controls } \\
\hline & Current smokers & Never-smokers & $p$-value ${ }^{a}$ & Current smokers & Never-smokers & $p$-value ${ }^{\mathrm{b}}$ \\
\hline $\mathrm{n}$ & 287 & 106 & & 109 & 235 & \\
\hline Female & $87(30.3)$ & $48(45.3)$ & 0.006 & $92(39.1)$ & $92(39.1)$ & 0.208 \\
\hline Age in years & $49.8 \pm 7.1$ & $52.1 \pm 6.6$ & 0.009 & $51.7 \pm 9.3$ & $52.4 \pm 11.1$ & 0.242 \\
\hline BMI $\left(\mathrm{kg} / \mathrm{m}^{2}\right)$ & $28.3 \pm 5.5$ & $29.1 \pm 5.9$ & 0.305 & $27.0 \pm 4.3$ & $27.0 \pm 4.6$ & 0.655 \\
\hline STEMI & $147(51.2)$ & $46(43.4)$ & 0.169 & - & - & - \\
\hline NSTEMI & $121(42.2)$ & $49(46.2)$ & 0.470 & - & - & - \\
\hline UA & $19(6.6)$ & $11(10.4)$ & 0.213 & - & - & - \\
\hline Diabetic & $33(11.5)$ & $20(18.9)$ & 0.058 & $8(7.3)$ & $16(6.8)$ & 0.857 \\
\hline Hypertensive & $165(57.5)$ & $68(64.2)$ & 0.233 & $60(55.0)$ & $135(57.4)$ & 0.676 \\
\hline HLA-DRB $1^{*} 01$ positive & $97(33.9)$ & $49(46.2)$ & 0.025 & $32(29.6)$ & $58(24.8)$ & 0.344 \\
\hline Asthma & $14(4.9)$ & $6(5.7)$ & 0.754 & $6(5.5)$ & $22(9.4)$ & 0.224 \\
\hline Cancer & $5(1.7)$ & $2(1.9)$ & 1.000 & $6(5.5)$ & $10(4.3)$ & 0.592 \\
\hline Rheumatoid arthritis & $10(3.5)$ & $9(8.5)$ & 0.060 & $2(1.8)$ & $7(3.0)$ & 0.725 \\
\hline $\mathrm{CRP}(\mathrm{mg} / \mathrm{l})$ & $13.1 \pm 19.6$ & $18.0 \pm 33.5$ & 0.490 & $2.9 \pm 5.7$ & $2.6 \pm 6.3$ & 0.031 \\
\hline TLC (10E9/ml) & $11.1 \pm 4.2$ & $9.0 \pm 3.3$ & $<0.001$ & - & - & \\
\hline $\mathrm{TC}(\mathrm{mmol} / \mathrm{l})$ & $4.8 \pm 1.2$ & $4.7 \pm 1.1$ & 0.885 & $5.5 \pm 0.9$ & $5.3 \pm 0.9$ & 0.054 \\
\hline HDL-C (mmol/l) & $1.2 \pm 0.4$ & $1.3 \pm 0.4$ & 0.058 & $1.4 \pm 0.4$ & $1.4 \pm 0.4$ & 0.922 \\
\hline $\mathrm{LDL}-\mathrm{C}(\mathrm{mmo} / \mathrm{l})$ & $2.7 \pm 1.0$ & $2.7 \pm 1.0$ & 0.992 & $3.4 \pm 0.9$ & $3.3 \pm 0.8$ & 0.201 \\
\hline TG $(\mathrm{mmol} / \mathrm{l})$ & $2.0 \pm 2.0$ & $1.7 \pm 1.0$ & 0.069 & $1.6 \pm 0.9$ & $1.4 \pm 0.8$ & 0.115 \\
\hline $\operatorname{TnT}(\mathrm{ng} / \mathrm{l})$ & $2.8 \pm 4.5$ & $2.6 \pm 3.4$ & 0.741 & - & - & - \\
\hline$\beta$-blockers & $79(62.7)$ & $32(58.2)$ & 0.566 & $19(17.4)$ & $39(16.6)$ & 0.847 \\
\hline ACE inhibitors & $37(37.8)$ & $14(31.1)$ & 0.441 & $9(8.3)$ & $22(9.4)$ & 0.739 \\
\hline ATR blockers & $20(23.5)$ & $10(24.4)$ & 0.915 & $7(6.4)$ & $17(7.2)$ & 0.783 \\
\hline Diuretic & $13(15.1)$ & $4(9.5)$ & 0.580 & $6(5.5)$ & $12(5.1)$ & 0.877 \\
\hline Lipid lowering therapy & $65(56)$ & $28(54.9)$ & 0.892 & $13(11.9)$ & $27(11.5)$ & 0.906 \\
\hline
\end{tabular}

Data is presented as mean \pm SD or number of study subjects (\%).

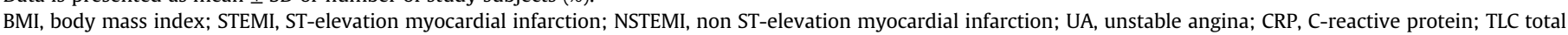

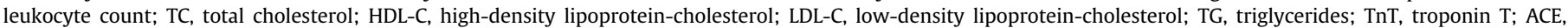
angiotensin-converting enzyme; ATR, angiotensin receptor.

$H L A-D R B 1^{*} 01$ positive $=1$ or 2 allele copies of $H L A-D R B 1^{*} 01$.

a Current smoking cases versus never-smoking cases.

b Current smoking controls versus never-smoking controls.

DR levels, especially in diseases known to be associated with smoking, and when the frequency of smokers differs between the study groups.

\subsection{SHLA-DR and smoking}

Cigarette smoking has both pro-inflammatory and immune suppressive influences on the immune system [19]. Smoking increases the risk of various autoimmune diseases with mechanisms which are still poorly understood $[20,21]$. It is a strong environmental risk factor for coronary artery disease as well as for rheumatoid arthritis [22,23].

To our knowledge, the previous studies of sHLA-DR have not assessed the associations between smoking and sHLA-DR levels.
Interestingly, alveolar macrophages of smokers have been observed to express less HLA-DR compared to alveolar macrophages of nonsmokers [24,25]. As the surface expression of HLA-DR has been shown to correlate with SHLA-DR [4], this could corroborate our findings. As our material seems to be nearly three times larger than any of the previous studies of sHLA-DR, the previous studies may also have been underpowered to detect such an association [1-8,10-12,26-34].

It is important to note that smoking explained only part of the variation in SHLA-DR levels and the strength of the correlation was modest. However, in cases, no other factor was identified with a significant correlation with sHLA-DR. In the control population, age, rheumatoid arthritis and $H L A-D R B 1^{*} 01$ carrier status were all associated with sHLA-DR. Even though the amount of smoking per 
day did not correlate with sHLA-DR, we think that the association between smoking and sHLA-DR was the most important association as i) it was seen both in case and in control populations ii) in the control population, smoking showed stronger correlation to sHLA-DR levels than other variables and iii) adjusting for smoking diminished the observed association between sHLA-DR levels and ACS in the binary logistic regression analyses.

\section{2. $s H L A-D R$ and inflammation}

Previous, smaller studies (ranging from 5 to 386 study subjects) have shown that SHLA-DR serum or plasma levels are elevated in patients with HLA class II-associated inflammatory disorders. Even though the $H L A-D R B 1^{*} 01$ was associated with ACS in the present, largest study of sHLA-DR (958 study subjects), we could not find an association between ACS and SHLA-DR. However, we were able to confirm the previously reported association of rheumatoid arthritis with higher sHLA-DR levels in our control population [8]. Other markers of inflammation or CRP were not associated with SHLA-DR levels. In controls, increasing age usually associated with increased levels of inflammation, exhibited inverse correlation with sHLA-DR.

In a previous study, sHLA-DR serum levels were higher in rheumatoid arthritis patients with disease-associated epitope, including $H L A-D R B 1^{*} 01$ and $H L A-D R B 1^{*} 04$ alleles, compared to rheumatoid arthritis patients without this disease-associated epitope [8]. In the present study, $H L A-D R B 1^{*} 01$ negative controls had higher serum levels of SHLA-DR than $H L A-D R B 1^{*} 01$ positive controls. By contrast, we did not observe any correlation between HLA-DRB $1^{*} 01$ allele positivity and sHLA-DR serum levels in cases. On the other hand, $H L A-D R B 1^{*} 01$ negative cases were current smokers with a significantly higher frequency compared to $H L A-$ $D R B 1^{*} 01$ positive cases, which might have affected the results.

We detected sHLA-DR levels $\geq 0.100 \mu \mathrm{g} / \mathrm{ml}$ and nearly half of our cases and controls had detectable levels of sHLA-DR in serum. By contrast in former studies the percentage of control subjects showing detectable levels of sHLA-DR in serum has ranged from 8 to $100 \%$. This is probably due to different quantification methods which complicates the comparison of sHLA-DR levels between different studies. Given the lack of established methods for sHLADR measurement, we performed validations to confirm our method.

\subsection{Limitations}

Our study has some limitations that should be considered. We were able to obtain only one serum sample from each study subject. Thus, we did not know whether the detected sHLA-DR level was the case's constant state or, for example, a temporary consequence of ACS. Our control population was also older than the case population. In addition, smoking data were self-reported, which made it possible for study subjects to misreport their smoking habits. Additionally, we did not have information of amount and duration of smoking in cases, which could have affected the results. We did not use statistical adjustments for multiple testing because we wanted to avoid to increase the probability of considering truly important associations as insignificant. We rather carefully described the methods we used and explained the motives we had behind the different analyses.

\subsection{Conclusions}

The results indicate for the first time that lower sHLA-DR levels are associated with smoking, but not independently associated with ACS. This is an important finding because the previous studies of sHLA-DR have not accounted for the possible associations between smoking and sHLA-DR levels. Further studies are required to confirm these novel results and explore the mechanisms behind the observed associations.

\section{Conflict of interest}

The authors declared they do not have anything to disclose regarding conflict of interest with respect to this manuscript.

\section{Financial support}

This study was financially supported by Ida Montin Foundation, Emil Aaltonen Foundation, Aarne Koskelo Foundation, Finnish Medical Foundation and Finnish Foundation for Cardiovascular research, and Helsinki University Central Hospital special government funds (EVO \#TYH2012209, \#TYH2014312, \#TYH2017250).

\section{Author contribution}

J.T set up the research method (sandwich ELISA), performed statistical analyses and drafted the manuscript, R.T took part in research protocol design and helped with statistical analyses and drafting the manuscript. H.J helped to set up the ELISA method and perform the data analyses. P.P helped with building up the ELISA method, V.S and A.H participated in data collection. M-L.L and J.S participated in research protocol design and data collection and supervised the whole study project. All authors critically revised the manuscript, read and approved the final manuscript.

\section{Acknowledgements}

The authors thank Seppo Meri for providing laboratory facilities and Lauri Snellman for participating in laboratory analyses.

\section{Appendix A. Supplementary data}

Supplementary data related to this chapter can be found at https://doi.org/10.1016/j.atherosclerosis.2017.09.023.

\section{References}

[1] D. Aultman, I. Adamashvili, K. Yaturu, M. Langford, F. Gelder, M. Gautreaux, et al., Soluble HLA in human body fluids, Hum. Immunol. 60 (1999) 239-244.

[2] R. Claus, T. Bittorf, H. Walzel, J. Brock, R. Uhde, D. Meiske, et al., High concentration of soluble HLA-DR in the synovial fluid: generation and significance in rheumatoid-like inflammatory joint diseases, Cell Immunol. 206 (2000) 85-100.

[3] Y.T. Ting, P.T. Coates, H.P. Marti, A.C. Dunn, R.M. Parker, J.W. Pickering, et al., Urinary soluble HLA-DR is a potential biomarker for acute renal transplant rejection, Transplantation 89 (2010) 1071-1078.

[4] M. Jendro, J.J. Goronzy, C.M. Weyand, Structural and functional characterization of HLA-DR molecules circulating in the serum, Autoimmunity 8 (1991) 289-296.

[5] M. Ott, C. Seidl, U. Westhoff, K. Stecker, E. Seifried, P.A. Fischer, et al., Soluble HLA class I and class II antigens in patients with multiple sclerosis, Tissue Antigens 51 (1998) 301-304.

[6] L.A. Verbruggen, H. Versaen, V. Rebmann, W. Duquet, S. De Cock, H. GrosseWilde, et al., Soluble HLA-DR levels in serum are associated with therapy and genetic factors in rheumatoid arthritis, Hum. Immunol. 63 (2002) 758-764.

[7] M. Hagihara, K. Hosoi, T. Kagawa, B. Gansuvd, B. Munkhbat, T. Shimura, et al., Serum soluble HLA-DR antigens in autoimmune hepatitis, Autoimmunity 31 (1999) 85-93.

[8] L.A. Verbruggen, N. Dumarey, H. Van de Velde, V. Rebmann, J. Flament, C. Van Wayenberge, et al., Soluble HLA-DR antigen levels in serum correlate with rheumatoid arthritis disease activity and the presence of disease-associated epitopes, Tissue Antigens 56 (2000) 436-440.

[9] C.M. Weyand, M. Jendro, J.J. Goronzy, Soluble HLA-DR molecules in patients with HLA class II versus class I associated disorders, Autoimmunity 8 (1991) $281-287$.

[10] J.C. McDonald, I. Adamashvili, J.M. Hayes, D.F. Aultman, V.K. Rhynes, F.B. Gelder, Soluble HLA class II concentrations in normal individuals and transplant recipients. Comparison with soluble HLA class I concentrations, 
Transplantation 58 (1994) 1268-1272.

[11] M. Ditschkowski, E. Kreuzfelder, V. Rebmann, S. Ferencik, M. Majetschak, E.N. Schmid, et al., HLA-DR expression and soluble HLA-DR levels in septic patients after trauma, Ann. Surg. 229 (1999) 246-254.

[12] V. Rebmann, S. Ugurel, W. Tilgen, U. Reinhold, H. Grosse-Wilde, Soluble HLADR is a potent predictive indicator of disease progression in serum from earlystage melanoma patients, Int. J. Cancer 100 (2002) 580-585.

[13] G. Liuzzo, J.J. Goronzy, H. Yang, S.L. Kopecky, D.R. Holmes, R.L. Frye, et al, Monoclonal T-cell proliferation and plaque instability in acute coronary syndromes, Circulation 101 (2000) 2883-2888.

[14] A. Palikhe, J. Sinisalo, M. Seppanen, V. Valtonen, M.S. Nieminen, M.L. Lokki, Human $\mathrm{MHC}$ region harbors both susceptibility and protective haplotypes for coronary artery disease, Tissue Antigens 69 (2007) 47-55.

[15] J. Sinisalo, E. Vlachopoulou, M. Marchesani, J. Nokelainen, M.I. Mayranpaa, J. Lappalainen, et al., Novel 6p21.3 risk haplotype predisposes to acute coronary syndrome, Circ. Cardiovasc Genet. 9 (2016) 55-63.

[16] S. Vaara, M.S. Nieminen, M.L. Lokki, M. Perola, P.J. Pussinen, J. Allonen, et al., Cohort profile: the corogene study, Int. J. Epidemiol. 41 (2012) 1265-1271.

[17] E. Vartiainen, T. Laatikainen, M. Peltonen, A. Juolevi, S. Mannisto, J. Sundvall, e al., Thirty-five-year trends in cardiovascular risk factors in Finland, Int. J. Epidemiol. 39 (2010) 504-518.

[18] R. Paakkanen, M.L. Lokki, M. Seppanen, I. Tierala, M.S. Nieminen, J. Sinisalo, Proinflammatory HLA-DRB1*01-haplotype predisposes to ST-elevation myocardial infarction, Atherosclerosis 221 (2012) 461-466.

[19] M.R. Stampfli, G.P. Anderson, How cigarette smoke skews immune responses to promote infection, lung disease and cancer, Nat. Rev. Immunol. 9 (2009) $377-384$.

[20] O. Vittecoq, T. Lequerre, V. Goeb, X. Le Loet, T.A. Abdesselam, N. Klemmer, Smoking and inflammatory diseases, Best. Pract. Res. Clin. Rheumatol. 22 (2008) 923-935.

[21] M. Sopori, Effects of cigarette smoke on the immune system, Nat. Rev. Immunol. 2 (2002) 372-377.

[22] T. Inoue, Cigarette smoking as a risk factor of coronary artery disease and its effects on platelet function, Tob. Induc. Dis. 2 (2004) 27-33.

[23] K. Chang, S.M. Yang, S.H. Kim, K.H. Han, S.J. Park, J.I. Shin, Smoking an rheumatoid arthritis, Int. J. Mol. Sci. 15 (2014) 22279-22295.

[24] W. Pankow, K. Neumann, J. Ruschoff, R. Schroder, P. von Wichert, Reduction in
HLA-DR antigen density on alveolar macrophages of smokers, Lung 169 (1991) 255-262.

[25] W. Pankow, K. Neumann, J. Ruschoff, P. von Wichert, Human alveolar macrophages: comparison of cell size, autofluorescence, and HLA-DR antigen expression in smokers and nonsmokers, Cancer Detect Prev. 19 (1995) $268-273$.

[26] A. Heiligenhaus, V. Rebmann, A. Neubert, S. Plewa, S. Ferencik, U. Vogeler, et al., Soluble HLA class I and HLA-DR plasma levels in patients with anterior uveitis, Tissue Antigens 63 (2004) 369-375.

[27] V. Rebmann, E. Ronin-Walknowska, O. Sipak-Szmigiel, A. Miklaszewicz, E. Czajkowska, H. Grosse-Wilde, Soluble HLA-DR and soluble CD95 ligand levels in pregnant women with antiphospholipid syndromes, Tissue Antigens 62 (2003) 536-541.

[28] M.W. Hayman, M.T. van Beijnen, L.K. Stamp, M.B. Spellerberg, J.L. O'Donnell, Soluble human leukocyte antigen: a diagnostic indicator of rheumatoid arthritis? J. Immunol. Methods 315 (2006) 19-26.

[29] L. Li, F. Kueppers, W. Hildebrand, R. Buchli, J. Gaughan, Elevated soluble HLA II protein levels in patients with alpha-1 antitrypsin deficiency with or without COPD, Exp. Lung Res. 38 (2012) 302-306.

[30] K.A. Pfeiffer, V. Rebmann, M. Passler, K. van der Ven, H. van der Ven, D. Krebs, et al., Soluble HLA levels in early pregnancy after in vitro fertilization, Hum. Immuno 161 (2000) 559-564.

[31] A. Higuchi, M. Hagihara, N. Tsumuraya, B. Gansuvd, S. Kato, T. Hotta, Comparison of soluble and membrane bound HLA-class I and DR levels in umbilical cord blood and adult peripheral blood, Tokai J. Exp. Clin. Med. 28 (2003) $1-7$.

[32] K. Hosoi, M. Hagihara, T. Kagawa, N. Watanabe, S. Matsuzaki, The serum soluble HLA-DR antigens as a predictive marker of the response to interferonalpha treatment in patients with chronic hepatitis C, Tokai J. Exp. Clin. Med. 25 (2000) 117-124.

[33] A. Steinborn, C. Sohn, V. Rebmann, H. Grosse-Wilde, Haemolysis, elevated liver enzymes, low platelet count (HELLP) syndrome associated with increased maternal serum levels of soluble HLA-DR antigens, Aust. N. Z. J. Med. 30 (2000) 511-512.

[34] A. Steinborn, V. Rebmann, A. Scharf, C. Sohn, H. Grosse-Wilde, Soluble HLA-DR levels in the maternal circulation of normal and pathologic pregnancy, Am. J. Obstet. Gynecol. 188 (2003) 473-479. 